\title{
ANÁLISE DAS FERRAMENTAS E MÉTODOS DE REDUÇÃO DO CUSTO ASSOCIADO À TOLERÂNCIA ESPECIFICADA DURANTE A FASE DE PROJETO DE UM COMPONENTE MECÂNICO
}

\author{
Cristiano Zeni (cristianozeni@ gmail.com) - PPGEM - UTFPR \\ Carlos Cziulik (cziulik@utfpr.edu.br)-DAMEC - UTFPR
}

\section{RESUMO}

A concorrência acirrada do mercado estimula as indústrias automotivas a percorrerem todos os caminhos para alcançarem a máxima satisfação dos clientes com o maior ganho econômico possível. Sendo assim, a redução de custos nas empresas possui papel fundamental. Uma das maneiras de eliminar desperdícios monetários é por meio da otimização dos valores das tolerâncias de projeto em componentes mecânicos, atividade esta, que não tem sido explorada em seu pleno potencial. Realizouse uma pesquisa com base literária e da experiência do autor, com o objetivo de avaliar como é conduzida a engenharia dimensional em uma empresa automotiva de grande porte. Constatou-se que os projetistas fazem uso majoritário da experiência e de conhecimentos adquiridos em projetos passados, afim de se alocar as tolerâncias com propriedade, o que causa uma nítida desvantagem nos desenvolvedores de produto com pouco tempo de prática. Normalmente o projetista tende a favorecer a qualidade, funcionalidade e robustez do componente, para evitar falhas e retrabalho. Além disso, outra prática bastante comum na indústria automotiva, ainda mais acentuada no ramo de caminhões $e$ ônibus, é a de, durante uma mudança em um componente, ou a criação de um novo, semelhante ao anterior, de não alterar nenhuma especificação de tolerância que não é afetada pela nova mudança. Este hábito é compreensivel, uma vez que garante que um componente ou função do componente, continuará a desempenhar seu papel satisfatoriamente. Examinando-se referências existentes, concluiu-se que apesar dos diversos estudos realizados dentro desse escopo, envolvendo análises focadas em perdas de manufatura, controle, qualidade, sustentabilidade e outras etapas do ciclo de vida de um produto, percebe-se que: devido à difícil aplicabilidade dos métodos, a aderência dos mesmos pela engenharia de produto ainda é reduzida. Tal efeito pode ser explicado à grande complexidade dos métodos envolvidos, da baixa adaptabilidade e da excessiva quantidade de dados de entrada necessários, que devem ser fornecidos pelo usuário. Desse modo, o objetivo do trabalho é a análise das ferramentas e bibliografia existentes para auxílio aos projetistas durante a fase de desenvolvimento, formulando questões e propondo melhorias para a criação futura de uma análise comparativa e qualitativa das tolerâncias, objetivando servir como guia para a alocação de valores, de maneira simples e rápida e visando a redução de custo.

\author{
Palavras chave: Tolerância. Custo de tolerâncias. Alocação de tolerâncias. Projeto de \\ produto.
}

Área: Gestão do Processo de Desenvolvimento de Produtos 


\section{INTRODUÇÃO}

A atribuição adequada de tolerâncias a peças mecânicas é uma atividade crítica dentro da fase de desenvolvimento do produto. Variações das dimensões críticas afetarão a qualidade, funcionalidade, custo e modularidade de um produto. Geralmente a seleção de tolerância é realizada levando em conta cálculos teóricos, experimentos e, principalmente, a experiência. Essas abordagens podem levar a vários equívocos e sobre dimensionamentos, pois é impossível para o projetista ou engenheiro considerar todos os aspectos relacionados ao custo, função, manufaturabilidade, manutenção, controle e confiabilidade que serão afetados por uma tolerância especificada. Além de várias ferramentas para auxiliar este processo, tais como: aprimoramento da disponibilidade de ferramentas de $\mathrm{CAD}$, métodos teóricos e práticos desenvolvidos na literatura e softwares específicos para cálculo de custo, a presença de reutilização de informação ou experiência de projetos passadas, continua a ser a principal abordagem atualmente utilizada.

A grande característica impactada de um dimensionamento incorreto ou impreciso, feito através da experiência, é o custo. Normalmente o projetista tende a favorecer a qualidade, funcionalidade e robustez do componente, visando evitar problemas futuros e retrabalho. Além disso, outra prática bastante comum na indústria automotiva, ainda mais acentuada no ramo de caminhões e ônibus, é a de, durante uma mudança em um componente, ou a criação de um novo bastante semelhante ao anterior, não se modificar nenhuma tolerância especificada que não é afetada pela nova mudança. Este hábito é compreensível, uma vez que garante que uma parte ou função da parte, que está funcionando corretamente hoje, continuará a funcionar satisfatoriamente. No entanto, escapa aos projetistas que essa tolerância poderia ter sido especificada há muito tempo, sem otimização adequada, selecionada e calculada sem as ferramentas que estão disponíveis atualmente, o que pode causar tolerâncias sobre especificadas, com grande custo intrínseco, que pode não estar contribuindo para qualquer qualidade ou melhoria funcional.

Uma situação comum nas empresas automotivas é o distanciamento do setor de desenvolvimento de produtos com a manufatura. Diferentes fornecedores, geralmente em diferentes cidades, estados e mais comumente, países, fabricam vários componentes projetados pela empresa principal. Esta distância tem grande impacto na comunicação e negociação dos pares, causando falta de feedback contínuo da experiência do fabricante para o projetista e viceversa. Não conhecer o verdadeiro estado da manufatura, suas dificuldades, possibilidades e fluxo de trabalho fará com que o alocador de tolerância perca possíveis otimizações em suas tolerâncias de peça.

Esse artigo visa traçar um perfil do toleranciamento na indústria mecânica, mostrando as soluções atuais para os problemas de custo de tolerâncias, com foco nas dificuldades dos projetistas em aplicá-las.

\section{A ESPECIFICAÇÃO DE TOLERÂNCIAS E SEUS IMPACTOS NO CUSTO DO PRODUTO}

A alocação de tolerâncias dimensionais é a abordagem primária para acomodar as variações resultantes dos sistemas de manufatura, dos processos de montagem, dos métodos de transporte e do uso, nas dimensões de um produto. A sua apropriada aplicação permite aos projetistas garantir um componente resistente o suficiente e com mínimo custo. Deve informar aos responsáveis pela fabricação e montagem da peça o quão preciso seu processo deve ser a fim 
de assegurar a qualidade mínima esperada pelo usuário final (SÖDERBERG; LINDKVIST; CARLSON, 2002).

A maior consequência financeira da escolha das tolerâncias é relacionada à precisão da manufatura. Afinal, a obtenção de uma peça com pouca variação dimensional é mais custosa e, além disso, todo produto com dimensões inaceitáveis deve ser refugado ou inutilizado. Porém, vários outros impactos são observados: a perda da satisfação e confiança do usuário do produto final, resultante da baixa qualidade de um produto, o impacto ecológico e socioambiental do descarte de produtos defeituosos, o custo de controle de dimensões excessivamente controladas e a baixa possibilidade de se reaproveitar os componentes em uma próxima atividade de remanufatura; entre outros. (HOFFENSON; SÖDERBERG, 2015).

Dentro do desenvolvimento do produto, a chamada "engenharia dimensional" é uma atividade importante para assegurar que os requisitos de projeto sejam atendidos, nele, uma maneira de controlar as variações e de se obter limites permissíveis para os componentes é a alocação de tolerâncias. Sua função principal é definir um requerimento funcional e, entendendo as origens das variações, seja no processo de manufatura, montagem ou variações externas, avaliar os impactos das mesmas e atribuir a correta tolerância dimensional (OMBASIC; ZARE, 2013).

Na manufatura, as tolerâncias de projeto são transformadas em tolerâncias de processo. Nessa etapa são calculados os efeitos de cada tolerância, além da capabilidade do componente (PAHL et al., 2007). Após, são selecionados os métodos de fabricação, outras otimizações de custos são desdobradas e diversas análises de montagem e fabricação são realizadas (CAMARGO; ABACKERLI, 2010).

Em paralelo à manufatura, o controle dimensional do componente é planejado. Determina-se o método de medição, definem-se as amostragens com base nas criticidades selecionadas no desenho e cria-se um plano de controle e validação da qualidade (CHASE et al., 1990).

Por fim, o produto a até o usuário final, com suas infinitas possibilidades de dimensões, o qual se espera estar dentro da conformidade definida. Ainda assim, a grande variação dessas especificações pode causar efeitos indesejáveis aos usuários, como: ruídos, vibração, desgaste, quebras e perda de eficiência. Cabe ao responsável pelo produto avaliar tais "inconsistências" e retornar com informações para os projetistas.

\section{A ALOCAÇÃO DE TOLERÂNCIAS NA FASE DE PROJETO}

O produto é desenvolvido e as especificações de produção do mesmo são consolidadas durante a fase conceitual. Nela, o dimensionamento é definido primeiramente com uma especificação robusta, tomando-se como base a experiência em outros projetos, ou o chamado design re-use (OMBASIC; ZARE, 2013). Tolerâncias são então alocadas no nível da peça considerando a sensibilidade geométrica, os custos de fabricação e o custo de qualidade (PENG, 2012). Após essa primeira etapa, geralmente tem-se o uso da análise estatística de tolerâncias para verificar a concepção do componente contra o sistema de produção assumido. Então, alocam-se as mesmas considerando as análises de otimização pertinentes.

Desenhos e modelos de engenharia são utilizados para a documentação de um projeto e para a efetiva comunicação entre indivíduos envolvidos em diferentes fases de um desenvolvimento. São as representações gráficas das intenções de um desenvolvedor, as quais apresentam as formas e dimensões de seu conceito, visando manter a integridade de uma peça manufaturada (HENZOLD, 1995). 
Para garantir que a informação seja assimilada de forma coerente e para eliminar ambiguidades na leitura de requerimentos, a indústria desenvolveu metodologias próprias para dimensionamento geométrico e toleranciamento funcional.

O propósito do toleranciamento funcional, segundo Anselmetti, (2006), é criar desenhos mecânicos de partes de um mecanismo de maneira a gerar geometrias aceitáveis utilizando um formato normalizado (ASTM, ISO). A base para a definição da funcionalidade de uma peça são seus requisitos funcionais. Ou seja, a condição física ou geométrica que deve ser respeitada a fim de possibilitar que a manufatura e montagem de um produto garantam plenamente o desempenho esperado do mesmo.

A especificação de tolerâncias depende demasiadamente da experiência em projetos anteriores. Todavia algumas literaturas são frequentemente utilizadas para direcionar a especificação, tais como:

a) Normas internacionais e nacionais, tais como a NBR 6158 (1995) e a ISO 286-1 (1990);

b) Normas internas, geralmente, disponibilizadas nas empresas de maior porte e baseadas em dados resultantes de desenvolvimentos de processos;

c) Literatura específica como, por exemplo, as obras de Bjorke, (1978) e Bralla (1997), que apresentam boas práticas para a criação do produto.

Outras ferramentas, métodos e boas práticas são utilizados no gerenciamento dimensional a fim de facilitar as atividades dos projetistas, as mais usuais são:

a) Formação de equipes de engenharia simultânea: que são organizadas no início do projeto e retidas até a sua conclusão, com o intuito de abordar todas as áreas impactadas e prover as informações necessárias. É, geralmente, composta de engenheiros de projeto, fabricação, qualidade e de membros com conhecimento ou experiência especializada. Muitas equipes também incluem representantes do cliente;

b) Diagramação dos objetivos, metas e interfaces: estabelecimento de requisitos de interface para que quaisquer alterações de engenharia relacionadas com o componente sejam mínimas.

c) DFM e DFA. O DFM tenta oferecer a compatibilidade entre a definição do produto e do processo de manufatura proposto. O objetivo geral é atender as capacidades de fabricação e processo para alcançar a intenção do projeto. Já o DFA foca em simplificar a montagem e reduzir custos e tempos de manufatura;

d) GD\&T. A especificação de tolerância geométrica segue um sistema internacional de representação em engenharia. Baseado em uma linguagem gráfica universalmente aceita, conforme normas nacionais e internacionais, busca melhorar a comunicação, o projeto de produto e a qualidade (XU; KEYSER, 2014);

e) Definição da característica chave. É uma característica de uma instalação, montagem ou detalhe do componente cuja variação dimensional tem o maior impacto no desempenho ou vida útil. A identificação das principais características de um produto específico é responsabilidade da equipe de gestão dimensional, em estreita ligação com o cliente.

f) Simulações de variação. Visam representar a realidade com simulações baseadas em conceitos estatísticos, tendo em conta a relação entre os requisitos funcionais, bem como o processo esperado e capacidades de medição.

Todavia, apesar das diversas ferramentas e métodos atuais, ainda se percebe falhas no dimensionamento de componentes e na especificação de tolerâncias na indústria. 


\section{MÉTODOS DE OTIMIZAÇÃO DE CUSTOS DE TOLERÂNCIAS}

Durante o século XX, vários métodos foram desenvolvidos visando limitar os custos resultantes da seleção inadequada de tolerâncias. Sejam eles para análises de cálculos de mínimo custo de manufatura, para cálculos de perda de qualidade e cálculos de custo de inspeção, sustentabilidade e impacto mercadológico. A grande maioria desses métodos possui complexidade elevada e aplicabilidade restrita (ISLAM, 2004), principalmente devido a serem quantitativos e absolutos. Ou seja, buscam valores globais de economia ao invés de realizar um comparativo qualitativo entre possíveis soluções.

Como as especificações de tolerância possuem influências nas diversas fases do desenvolvimento do produto, muitas análises diferentes foram conduzidas, desde o custo mínimo para manufatura, até o custo de sustentabilidade ambiental e mercadológica. Decidiuse agrupar os métodos em quatro grandes áreas:
a) Manufatura;
b) Qualidade;
c) Controle;
d) Híbridos.

As duas primeiras são as que apresentam a maior quantidade de referências, visto que são as mais impactantes nos custos, e serão as detalhadas no artigo.

\subsection{Alocação de Tolerâncias para Mínimo Custo de Manufatura}

Tolerâncias restritas levam a custos excessivos de fabricação, enquanto especificações muito flexíveis podem gerar desperdício excessivo e problemas de montagem e qualidade (PUROHIT; SHARMA, 1999).

Autores como Evans (1958) e Pike e Silverberg. (1953) foram os pioneiros no desenvolvimento de teorias e formulações matemáticas no assunto, Já Chase (1999) sumarizou os estudos anteriores e mostrou que para a alocação de tolerância de custo mínimo, é necessário caracterizar o relacionamento matemático dos valores de tolerância com o custo das mesmas, a fim de selecionar qual combinação é a mais rentável. No exemplo mostrado na Figura 1, cada método de fabricação apresenta um custo $(\mathrm{C})$ determinado para as tolerâncias $(\mathrm{T})$.

Figura 1 - Custo de tolerâncias para processos de fabricação especificados. Fonte: Adaptado de Chase (1999).

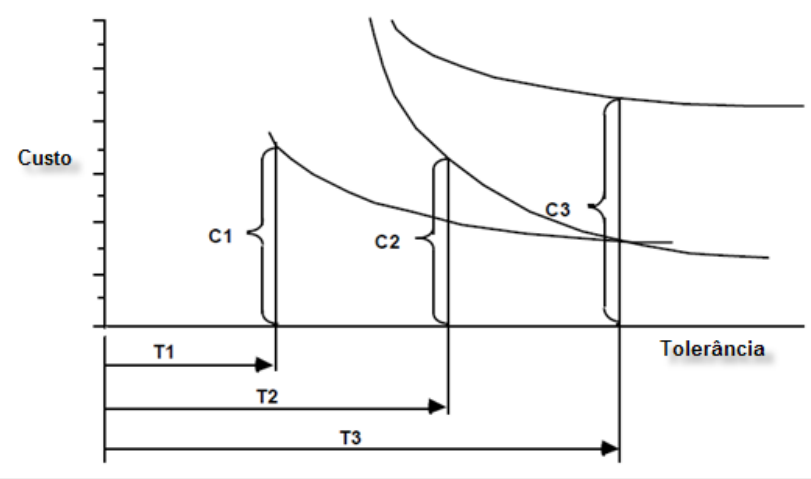

Apesar de simples e matematicamente robusta essa abordagem não foi amplamente utilizada devido à grande dificuldade de extrair tais funções de maneira empírica. O número de variáveis 
presentes para a determinação é volumoso. Qualquer variação no ambiente, como grau de afiação da ferramenta, temperatura, máquina e calibração, podem gerar uma variação no custo difícil de ser precisada.

Outra grande dificuldade no tratamento do problema envolvendo custos e tolerâncias de fabricação é sua dependência das condições locais de manufatura. A maioria das empresas e fornecedores não disponibiliza tais dados. Um fator que influencia favoravelmente no custo real ou absoluto de um componente é sua escala de produção. O custo do material e de insumos é, geralmente, menor para uma maior quantidade produzida devido a fatores de negociação comercial. Também, o processo é ajustado para um maior tamanho de lote. Ou seja, o custo relacionado ao tempo de ajuste do processo (setup) é rateado por um número maior de peças.

Visto essas dificuldades, alguns autores partiram para a análise mais comparativa e qualitativa do custo em tolerâncias, como os mostrados a seguir.

Dimitrellou; Diplaris e Sfantsikopoulos, (2006) criaram uma planilha de análise com fatores genéricos de avaliação, tendo o objetivo de comparar cada categoria de um elemento de tolerância conforme sua habilidade de ser produzido. O método se baseia no preenchimento de um formulário, pela manufatura, com valores representativos para cada classe de tolerância IT. Baseado na opinião da manufatura e usando a tabela preenchida como banco de dados, era criado um método de otimização que buscava definir o conjunto de tolerâncias, com menor impacto na fabricação.

Essa análise simplificou consideravelmente o processo de otimização, porém ainda requer um tempo de setup muito grande para poder ser usado imediatamente. Em uma empresa de grande porte, com componentes de materiais diversos, e, com uso de diversos métodos de fabricação diferentes, o seu uso fica restrito ao preenchimento de um número elevado de campos. Em uma empresa automotiva, por exemplo, seria necessário exigir o preenchimento da planilha para cada fornecedor, dentre os inúmeros da companhia.

O modelo proposto por Sanz-lobera; Sebastián e Jesus (2010) é fundado sobre o estabelecimento de uma relação de custo-tolerância individualizada considerando a variabilidade existente de recursos de produção a cada momento. O modelo calcula o número de peças que estão fora da especificação e subsequentemente, o número de peças adicionais que devem ser fabricadas para compensar essa perda.

Sivakumar; Balamurugan e Ramabalan (2011) foram pioneiros em juntar o cálculo de otimização de custo de tolerâncias com a seleção de processos. Ambos os assuntos eram previamente tratados de maneira separada. Em seu estudo, de grande complexidade computacional, desenvolveram um conjunto de equações e sistemas e os incluíram em uma rotina de otimização. Não desenvolveram uma interface para o usuário, e devido à grande capacidade computacional necessária para execução, as analise ficam restritas a problemas simples.

Percebe-se pela grande quantidade de referências existentes, que poucas conseguem, de maneira eficaz, prover um método simples, rápido e que forneça informações de valia para os projetistas, quanto ao custo de manufatura relacionado a tolerâncias.

\subsection{Alocação de Tolerâncias para Mínimo Custo de Qualidade}

A engenharia de qualidade usa o projeto de um produto como meio para aumentar a confiabilidade dos componentes, predominantemente pela redução da variância das especificações. Basicamente existem dois modos de examinar os requisitos, a fim de reduzir a variação de fabricação de um produto. O primeiro, pela mudança do valor nominal de uma 
dimensão, alteração essa que, geralmente, não afeta os custos. O segundo é pela alteração específica da tolerância, que como discutido anteriormente, gera custo. Portanto, é sempre preferível mudar os parâmetros nominais de desempenho às tolerâncias, por motivos econômicos.

No ramo de toleranciamento para aperfeiçoamento de qualidade, se destaca Taguchi; Elsayed e Hsiang (1989), que iniciaram o estudo desse ramo e criaram uma função de perda de qualidade para variações de tolerâncias. Propuseram que a perda de qualidade deve ser tratada como uma despesa, juntamente com o custo de fabricação. Esta medida de perda de qualidade representa a perda para a sociedade que ocorre quando um produto se desvia do conjunto ótimo de parâmetros de concepção. Os desvios são controlados por tolerâncias. A função perda de qualidade transforma a degradação em um custo, que podem então ser incluídos em uma função objetivo, juntamente com os custos de produção. A função quadrática, mostrada na Figura 2, pode prever uma estimativa para o detrimento de qualidade segundo a característica do produto. A perda é maior que zero se a característica funcional desvia da sua dimensão tida como meta, não importando o tamanho da oscilação.

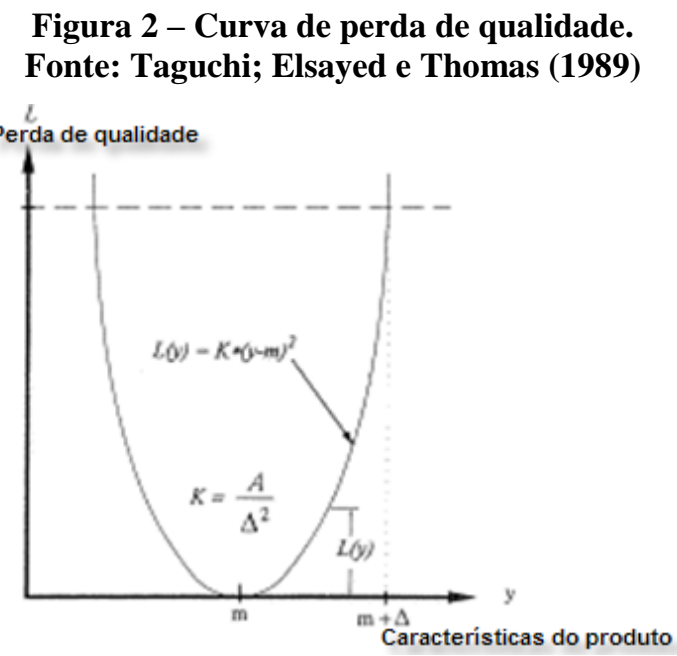

A partir dos estudos de Taguchi; Elsayed e Hsiang (1989), diversos autores desenvolveram métodos próprios para a resolução do custo de perda de qualidade para tolerâncias, como: (JEANG, 2011; YE; SALUSTRI, 2003; ZHANG; YANG, 2009).

Vale ressaltar que a grande maioria dos estudos foi conduzido com base em uma formulação matemática pesada e complexa, tendem a aperfeiçoar com precisão o custo para a síntese de tolerâncias e os métodos aplicados assumem que o componente que será tolerado é novo e com características funcionais independentes.

\subsection{Métodos Híbridos de Toleranciamento para Mínimo Custo}

Com o maior uso da engenharia simultânea, a quantidade de informação necessária para o perfeito desenvolvimento de um componente em todos os estágios de criação de um produto é cada vez maior. A grande competitividade do mercado levou à popularização do chamado "projeto para $\mathrm{x}$ " (onde se entende por " $\mathrm{x}$ " qualquer ramo impactado pelo design, pode ser entendido como: manufatura, qualidade, mantenabilidade, custo, entre outros). Tais tendências iniciaram uma nova fase do estudo do toleranciamento para mínimo custo. Uma fase que pretende, com o avanço da pesquisa no ramo de toleranciamento, com modelos cada vez mais sofisticados e tirando grande proveito do poder computacional atual e principalmente, com o 
auxílio das ferramentas de CAD, CAM e CAE, criar análises de otimização simultâneas para diferentes geradores de custo.

Com os estudos de Taguchi; Elsayed e Hsiang (1989) percebeu-se que um aumento do valor de uma tolerância pode causar impactos tanto positivos quanto negativos no custo de um produto. Tolerâncias apertadas são preferidas para garantir a performance e evitar custos de qualidade. Porém, implicam em um alto custo de manufatura. Essa relação conflitante foi o início para os primeiros estudos nessa área e para os primeiros métodos híbridos, ou seja, que consideram em uma mesma análise a melhoria de custo de fabricação e de qualidade. Os mais relevantes para o trabalho em questão são descritos na Tabela 1.

Tabela 1 - Sumário de análise de otimização de custos de tolerâncias (híbridos). Fonte: Autoria Própria.

\begin{tabular}{|c|c|c|c|c|}
\hline $\begin{array}{l}\text { Método / } \\
\text { Estudo } \\
\end{array}$ & Particularidade & $\begin{array}{c}\text { Forma de } \\
\text { Análise }\end{array}$ & Vantagem & $\begin{array}{c}\text { Desvantag } \\
\text { em }\end{array}$ \\
\hline $\begin{array}{c}\text { Wu; Chen e Tang } \\
(1998)\end{array}$ & $\begin{array}{c}\text { Primeira a fazer a } \\
\text { análise. }\end{array}$ & \multirow{3}{*}{ Gráfica } & \multirow{3}{*}{$\begin{array}{l}\text { Facilidade de uso e } \\
\text { entendimento. } \\
\text { Simples de } \\
\text { generalizar }\end{array}$} & \multirow{3}{*}{$\begin{array}{l}\text { Análises } \\
\text { precisas e de } \\
\text { otimização } \\
\text { são muito } \\
\text { limitadas }\end{array}$} \\
\hline Umaras (2010) & $\begin{array}{l}\text { Descreve melhor as } \\
\text { fórmulas e conceitos. }\end{array}$ & & & \\
\hline $\begin{array}{c}\text { Chen e } \\
\text { Maghsoodloo } \\
\text { (1995) }\end{array}$ & $\begin{array}{l}\text { Inclusão de fatores } \\
\text { estatísticos e lógicos } \\
\text { para melhorar } \\
\text { qualidade da análise. }\end{array}$ & & & \\
\hline Choi et al., (2000), & $\begin{array}{l}\text { Primeiro a utilizar } \\
\text { formulaçõos } \\
\text { matemáticas } \\
\text { complexas. } \\
\end{array}$ & \multirow{2}{*}{$\begin{array}{l}\text { Multiplicadores de } \\
\text { LaGrange; }\end{array}$} & \multirow{2}{*}{$\begin{array}{c}\text { Fórmulas } \\
\text { matemáticas } \\
\text { complexas. Difícil de } \\
\text { generalizar. }\end{array}$} & \multirow{2}{*}{$\begin{array}{l}\text { Análises } \\
\text { mais } \\
\text { precisas. }\end{array}$} \\
\hline Peng, (2012) & $\begin{array}{l}\text { Incluiu a análise de } \\
\text { perda de qualidade } \\
\text { com o tempo } \\
\text { (degradação). }\end{array}$ & & & \\
\hline $\begin{array}{c}\text { Lee e Johnson, } \\
\text { (1993) } \\
\text { Shan et al, (2003) } \\
\text { Al-Ansary e Deiab } \\
\text { (1997) }\end{array}$ & $\begin{array}{l}\text { Uso de grande } \\
\text { capacidade } \\
\text { computacional }\end{array}$ & $\begin{array}{l}\text { Algoritmos } \\
\text { genéticos }\end{array}$ & $\begin{array}{c}\text { Exige o } \\
\text { desenvolvimento de } \\
\text { algoritmo complexo } \\
\text { específico para cada } \\
\text { caso estudado, sendo } \\
\text { de difícil aplicação } \\
\text { prática. }\end{array}$ & $\begin{array}{l}\text { Precisão de } \\
\text { análise em } \\
\text { alto nível, } \\
\text { para o caso } \\
\text { específico. }\end{array}$ \\
\hline
\end{tabular}

O detalhamento de cada análise não é descrito aqui, mas pode-se sumarizar que são de aplicabilidade restrita, possuem grande dependência de diversas variáveis que são difíceis de se coletar com precisão necessária e principalmente, são baseadas em modelos matemáticos altamente sensíveis a variações.

\section{CONSIDERAÇÕES FINAIS}

Como se pôde observar, a atribuição de tolerâncias é uma linha de pesquisa abrangente. Normas, métodos, modelos e metodologias diversas existem afim de explorar todos os detalhes e impactos do assunto. Nota-se, que repercussão da correta seleção dos valores não é apenas restrita à funcionalidade do produto mas, também, ao seu custo e a todas as etapas do seu ciclo de vida. 
Realizando-se uma análise crítica dos estudos que visaram a otimização de custos de tolerância, percebe-se que prevalecem os referentes ao custo de manufatura, à perda de qualidade e à junção de ambos, os denominados híbridos. Predominantemente, há formulações matemáticas complexas e, restrições às aplicações específicas. Um pequeno número de estudos desenvolveu métodos qualitativos e tentou simplificar a análise. Pode-se afirmar que a abrangência desses métodos no mercado é reduzida também devido a esses fatores.

\section{REFERÊNCIAS}

AL-ANSARY, M. D.; DEIAB, I. M. Concurrent optimization of design and machining tolerances using the genetic algorithms method. International Journal of Machine Tools and Manufacture, v. 37, n. 12, p. 1721-1731, 1997.

ANSELMETTI, B. Generation of functional tolerancing based on positioning features. Computer-Aided Design, v. 38, n. 8, p. 902-919, 2006.

BJØRKE, Øyvind. Computer-aided tolerancing. Tapir, 1978.

BRALLA, James G. Design for manufacturability handbook. McGraw-Hill, 1999.

CAMARGO, R; ABACKERLI, A. importância da tolerância: uma contribuição da engenharia dimensional no processo de desenvolvimento do produto. Revista Ciência e Tecnologia, v. 11, n. 19, 2010.

CHASE, K. W. Minimum cost tolerance allocation. Department of Mech. Engg., Bringham Young University,. ADCATS Report, n. 99-5, 1999.

CHASE, K. W. et al. Least cost tolerance allocation for mechanical assemblies with automated process selection. Manufacturing review, v. 3, n. 1, p. 49-59, 1990.

CHOI, H. R.; PARK, M.; SALISBURY, E. Optimal tolerance allocation with loss functions. Journal of Manufacturing Science and Engineering, v. 122, n. 3, p. 529-535, 2000.

DIMITRELLOU, S. Ch; DIPLARIS, S. C.; SFANTSIKOPOULOS, M. M. Cost-competent tolerancing in CAD. The International Journal of Advanced Manufacturing Technology, v. 35, n. 5-6, p. 519-526, 2007.

HENZOLD, G. Handbook of Geometrical Tolerancing: Design, Manufacturing, and Inspection. John Wiley \& Sons, 1995.

HOFFENSON, S.; SÖDERBERG, Rikard. Systems thinking in tolerance and quality-related design decision-making. Procedia CIRP, v. 27, p. 59-64, 2015.

ISLAM, M. N. Functional dimensioning and tolerancing software for concurrent engineering applications. Computers in Industry, v. 54, n. 2, p. 169-190, 2004.

INTERNATIONAL ORGANIZATION FOR STANDARDIZATION, DIN. 286-1: ISOSystem für Grenzmaße und Passungen. Grundlagen für Toleranzen, Abmaße und Passungen, 2010.

JEANG, A.. Tolerance chart balancing with a complete inspection plan taking account of manufacturing and quality costs. The International Journal of Advanced Manufacturing Technology, v. 55, n. 5-8, p. 675-687, 2011.

LEE, J.; JOHNSON, G. E. Optimal tolerance allotment using a genetic algorithm and truncated Monte Carlo simulation. Computer-Aided Design, v. 25, n. 9, p. 601-611, 1993. 
OMBASIC, N.; ZARE, D. Dimensional engineering: methods and tools for a dimensional engineering process at scania, Chalmers University Report, 2013.

PAHL, G. et al. Engineering Design: A Systematic Approach. Engineering Design: A Systematic Approach, Edited by G. Pahl, W. Beitz, J. Feldhusen, and K.-H. Grote. Berlin: Springer, 2007 ISBN: 978-1-84628-318-5, v. 1, 2007.

PENG, $\mathrm{H}$. Concurrent tolerancing for design and manufacturing based on the present worth of quality loss. The International Journal of Advanced Manufacturing Technology, v. 59, n. 912, p. 929-937, 2012.

PIKE, E. W.; SILVERBERG, T. R. Assigning tolerances for maximum economy. Machine design, v. 25, p. 139, 1953.

PUROHIT, K.; SHARMA, C. S. Recent developments in tolerancing techniques. Defence Science Journal, v. 49, n. 4, p. 291, 1999.

SANZ-LOBERA, A.; SEBASTIÁN, M. A.; PÉREZ, J. M. New cost-tolerance model for mechanical part design. The International Journal of Advanced Manufacturing Technology, v. 51, n. 5-8, p. 421-430, 2010.

SHAN, A.; ROTH, R. N.; WILSON, R. J. Genetic algorithms in statistical tolerancing. Mathematical and computer modelling, v. 38, n. 11, p. 1427-1436, 2003.

SIVAKUMAR, K.; BALAMURUGAN, C.; RAMABALAN, S. Simultaneous optimal selection of design and manufacturing tolerances with alternative manufacturing process selection. Computer-Aided Design, v. 43, n. 2, p. 207-218, 2011.

SÖDERBERG, R.; LINDKVIST, L; CARLSON, Johan. Virtual geometry assurance for effective product realization. In: First Nordic Conference on Product Lifecycle ManagementNordPLM. 2006. p. 25-26.

TAGUCHI, G.; ELSAYED, Elsayed A.; HSIANG, T. C. Quality Engineering in Production Systems. McGraw-Hill College, 1989.

UMARAS, E. Tolerâncias dimensionais em conjuntos mecânicos: estudo e proposta para otimização. 2010, 75 f. Dissertação (Mestrado) - Universidade de São Paulo, 2010.

XU, S.; KEYSER, J. Geometric computation and optimization on tolerance dimensioning. Computer-Aided Design, v. 46, p. 129-137, 2014.

YE, B.; SALUSTRI, F. A. Simultaneous tolerance synthesis for manufacturing and quality. Research in Engineering Design, v. 14, n. 2, p. 98-106, 2003.

ZHANG, Y.; YANG, M. A coordinate SPC model for assuring designated fit quality via quality-oriented statistical tolerancing. Computers \& Industrial Engineering, v. 57, n. 1, p. 73-79, ago. 2009. 